\title{
原位自发泡制备磷酸钲/聚氨酯复合骨修复支架
}

\author{
李根, 李昫昫, 李丽梅, 蒋佳兴, 李玉宝, 李吉东 \\ (四川大学 纳米生物材料研究中心, 分析测试中心, 成都 610064)
}

摘 要: 磷酸钙/聚氨酯( $\mathrm{CaP} / \mathrm{PU})$ 复合骨修复支架制备过程中随着材料体系粘度逐渐增大, 后期加入的发泡剂难于 均匀分散, 影响支架孔隙率及孔结构均匀性。本研究在 $\mathrm{CaP} / \mathrm{PU}$ 材料合成过程中将发泡剂水以磷酸氢钙结晶水合物 (DCPD)的形式均匀复合在材料中, 在一定条件下释放结晶水与聚氨酯(PU)中的异氧酸根反应生成 $\mathrm{CO}_{2}$, 实现自发 泡成型。实验结果显示, $90{ }^{\circ} \mathrm{C}$ 条件下自发泡制备的 $\mathrm{CaP} / \mathrm{PU}$ 支架孔隙率高、孔结构均匀、贯通性好。将 $90{ }^{\circ} \mathrm{C}$ 发泡 成型的 $\mathrm{CaP} / \mathrm{PU}$ 多孔支架在 $110{ }^{\circ} \mathrm{C}$ 再熟化处理，可提高支架的力学性能高达 1 倍以上。该方法简便易行，为聚氨酯 基多孔支架的制备提供了新思路。

关 键 词: 磷酸氢钻; 聚氨酯; 骨修复; 支架; 自发泡

中图分类号: TB33 文献标识码: A

\section{Preparation of Calcium Phosphate/Polyurethane Composite Porous Scaffolds for Bone Repair by in situ Self-foaming Method}

\author{
LI Gen, LI Jiong-Jiong, LI Li-Mei, JIANG Jia-Xing, LI Yu-Bao, LI Ji-Dong \\ (Research Center for Nano-Biomaterial, Analytical \& Testing Center, Sichuan University, Chengdu 610064, China)
}

\begin{abstract}
One of the main technical problems in preparation of calcium phosphate/polyurethane (CaP/PU) composite scaffold for bone repair is to obtain a porous structure with uniformly distributed pores. The reason for this is due to the heterogeneous dispersion of the added foaming agents within the composite resulting in the increasing viscosity of polymer precursor during the composite fabrication process. To solve this problem, we report a novel method through incorporation of calcium hydrogen phosphate (DCPD) with polymer phase to achieve a composite system in early period of the synthesis process of $\mathrm{CaP} / \mathrm{PU}$ composite. The uniformly distributed crystal water from DCPD can be released when temperature reaches above $75{ }^{\circ} \mathrm{C}$ and serve as foaming agents to react with isocyanate group in the $\mathrm{PU}$ and generate $\mathrm{CO}_{2}$ gas. The generation of gas leads to a self-foaming process and consequently induces $\mathrm{CaP} / \mathrm{PU}$ composite to form a porous scaffold. Scanning electron microscopy (SEM) observations show that $\mathrm{CaP} / \mathrm{PU}$ composite scaffolds with uniform porous structure, high porosity and interconnectivity were obtained at $90{ }^{\circ} \mathrm{C}$. The mechanical strength can be doubled by re-curing treatment for the scaffold at $110{ }^{\circ} \mathrm{C}$ for $24 \mathrm{~h}$. The resulting $\mathrm{CaP} / \mathrm{PU}$ composite scaffolds with uniformly porous structure, high porosity and interconnectivity have shown potential applications in bone tissue engineering. Moreover, this simple and efficient method may provide new approaches for the preparation of polyurethane- based porous scaffolds.
\end{abstract}

收稿日期: 2015-12-14; 收到修改稿日期：2016-01-26

基金项目: 国家高技术研究发展计划重点项目(863 计划)(2013AA032203); 国家自然科学基金(31370971); 四川大学优秀青 年学者基金(2014SCU04A20)

National High Technology Research and Development Program of China (863) (2013AA032203); National Natural Science Foundation of China (31370971); Outstanding Young Scholar Fund of Sichuan University (2014SCU04A20)

作者简介: 李 根(1991-), 男, 硕士研究生. E-mail: 1536399719@qq.com

通讯作者: 李吉东, 副教授. E-mail: nic1979@scu.edu.cn 
Key words: calcium hydrogen phosphate; polyurethane; bone repair; scaffold; self-foaming

无机/有机复合多孔支架作为物质和结构模板, 在骨组织工程中发挥着重要作用 ${ }^{[1-2]}$ 。适宜的仿生孔 隙结构是多孔支架能否发挥最优成骨效能的关键 ${ }^{[3]}$ 。 尽管多孔支架已在骨组织工程研究和应用中取得了 较大进展, 但普遍存在力学性能、降解性能与自然 骨再生不匹配, 孔径不均匀和孔隙连通性差等问题, 使细胞和组织难以渗透到支架内部, 只在支架外围 生长, 同时物质运输受阻, 不利于局部组织更新, 难以满足临床应用需求 ${ }^{[4-5]}$ 。

骨组织主要是由磷酸钙矿物和胶原构成的天然 复合材料, 基于仿生理念制备的磷酸钙盐/聚合物仿 生复合材料显示出良好的生物活性和生物相容性 ${ }^{[6-7]}$ 。 聚氨酯是由硬段和软段构成的嵌段聚合物, 根据不 同需求选择不同的软硬段或调整软硬段比例即可获 得不同的理化性能和降解性能, 因其良好的生物相 容性和性能可灵活调控等优点已在组织工程领域得 到广泛关注 ${ }^{[8-9]}$ 。磷酸钲盐/聚氨酯 $(\mathrm{CaP} / \mathrm{PU})$ 复合组织 工程骨支架已显示出良好的应用潜力 ${ }^{[10-12]}$ 。因合成 聚氨酯原料中存在的异氧酸根可与水反应生成 $\mathrm{CO}_{2}$,

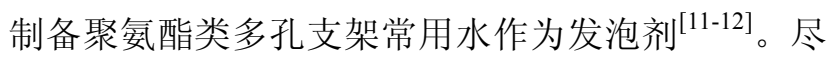
管水安全无毒, 但随着反应进行, 材料体系粘度逐 渐增大, 后期加入的水很难均匀混入其中, 从而影 响支架孔隙结构均匀性和孔隙连通性, 进而影响支 架力学等方面的性能。所以制备具有良好力学性能 的均匀多孔结构的磷酸钙盐/聚氨酯 $(\mathrm{CaP} / \mathrm{PU})$ 复合 组织工程骨支架仍需进一步探索。

磷酸氢钻结晶水合物 $\left(\mathrm{CaHPO}_{4} 2 \mathrm{H}_{2} \mathrm{O}, \mathrm{DCPD}\right)$ 常 用作磷酸钻骨水泥的前驱物, 具有良好的生物活性 和生物安全性 ${ }^{[13]}$ 。本研究针对上述发泡剂水难以均 匀分散问题, 以磷酸钙盐结晶水的形式均匀复合在 $\mathrm{CaP} / \mathrm{PU}$ 复合材料中, 并在一定条件下释放结晶水, 以 提供发泡剂在发泡过程中所需的水, 与 PU 中的异氰 酸根反应生成 $\mathrm{CO}_{2}$, 实现复合材料自发泡成型, 制备孔 隙均匀、贯通性好、力学性能佳的 $\mathrm{CaP} / \mathrm{PU}$ 多孔支架。

\section{1 实验方法}

\section{1 试剂与原料}

菎麻油 $(\mathrm{CO}) 、 1,4-$ 丁二醇 $(\mathrm{BDO}) 、$ 辛酸亚锡购 自成都科龙试剂厂, 异佛尔酩二异氰酸酯(IPDI)、甘 油 GL购自上海阿拉丁试剂有限公司, DCPD 购自天 津市光复精细化工研究所, 均为分析纯。

\section{2 磷酸钲/聚氨酯复合支架制备}

基本按李丽梅等 ${ }^{[14]}$ 报道的方法制备, 但以 DCPD 代替文献中的纳米羟基磷灰石(nHA), 制备 多孔磷酸氢钙/聚氨酯(DCPD/PU)复合骨修复支架, 其中无机填料约占 $30 \mathrm{wt} \%$ 。即在氮气保护下，取甘 油改性的菎麻油(GCO) $15 \mathrm{~g}$ 加入三颈瓶中, 油浴加 热至 $70{ }^{\circ} \mathrm{C}$, 保持一定强度的机械搅拌条件下, 加 入 DCPD 粉末(过 300 目笁) $13 \mathrm{~g}$, 使其与菎麻油充 分混合。逐渐滴加 $15 \mathrm{~g}$ IPDI, (蓖麻油与 IPDI 的摩 尔比为 $1: 1.5)$, 反应得到 $\mathrm{DCPD} / \mathrm{PU}$ 复合材料预聚 物。然后加入 $0.05 \mathrm{~mL}$ 催化剂辛酸亚锡反应 $0.5 \mathrm{~h}$, 再加入 $0.5 \mathrm{~mL}$ 扩链剂 1,4-丁二醇(BDO)继续反应 $2 \mathrm{~h}$, 出料分四等份, 分别在 $80{ }^{\circ} \mathrm{C} 、 90{ }^{\circ} \mathrm{C} 、 100{ }^{\circ} \mathrm{C}$ 和 $110{ }^{\circ} \mathrm{C}$ 熟化发泡 $24 \mathrm{~h}$, 洗涤、烘干得到 $\mathrm{CaP} / \mathrm{PU}$ 复合多孔支架。优选出最佳熟化发泡温度后, 以 $\mathrm{nHA}$ 和 DCPD 的混合粉末代替上述方法中的 $\mathrm{DCPD}$, 制备 nHA-DCPD/PU 复合多孔支架, 其中 $\mathrm{nHA}$ 按文献方法制得 ${ }^{[14]}$ 。为了进一步提高支架的 力学性能, $90{ }^{\circ} \mathrm{C}$ 条件下成型的支架再放入 $110{ }^{\circ} \mathrm{C}$ 烘箱继续熟化 $24 \mathrm{~h}$ 。所制备的复合支架的编号及成 分如表 1 所示。图 1 为聚氨酯聚合反应示意图及反 应中各成分的分子结构。

\section{3 表征方法}

采用扫描电子显微镜(SEM, JSM-6500LV, Jeol, Japan) 观察支架形貌, 傅里叶变换红外光谱仪(FTIR, Nicolet 6700, USA)分析材料官能团的位置和强度变 化, X 射线衍射仪 $(X R D$, 中国丹东方圆公司 DX2000 型)分析材料中无机粒子的晶相, 采用 AGIC50 型(SHIMADZU, Japan)力学试验机，根据国标 GB/T1041-92 测试支架的抗压强度和弹性模量, 试验压头速度为 $0.5 \mathrm{~mm} / \mathrm{min}$ 。采用同步热分析仪 (STA449F3，NETZSCH，Germany)分析支架的热稳 定性。

表 1 复合支架的编号及成分

Table 1 Abbreviation and composition of fabricated composite scaffolds

\begin{tabular}{cccc}
\hline Samples & $\begin{array}{c}\mathrm{nHA} \\
\text { percentage } \\
/ \mathrm{wt} \%\end{array}$ & $\begin{array}{c}\text { DCPD } \\
\text { percentage } \\
/ \mathrm{wt} \%\end{array}$ & $\begin{array}{c}\text { PU percentage } \\
/ \mathrm{wt} \%\end{array}$ \\
\hline DCPD/PU & 0 & 30 & 70 \\
nHA-DCPD/PU-1 & 15 & 15 & 70 \\
nHA-DCPD/PU-2 & 20 & 10 & 70 \\
\hline
\end{tabular}


<smiles>CC1(C)CC(C(=O)[O-])CC(C)(CN=O)C1</smiles>

IPDI

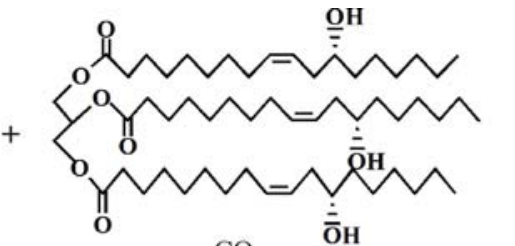

$\mathrm{CO}$

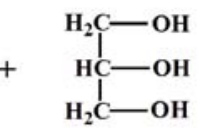

GL

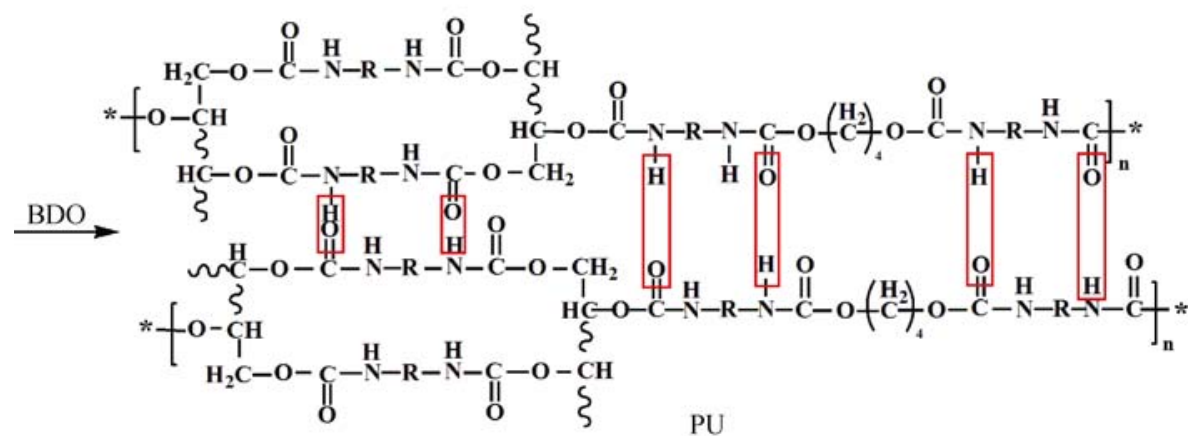

图 1 聚氨酯聚合反应示意图

Fig. 1 Synthetic scheme of polyurethane

\section{4 孔隙率测定与孔径分布统计}

采用水浸渍法测定孔隙率 ${ }^{[15]}$ : 干燥待测样品, 测量体积为 $V$, 称重 $\left(W_{1}\right)$; 然后浸入去离子水(密度 $\rho$ ) 中直至充盈浸透, 取湿样称重 $\left(W_{2}\right)$, 由公式(1)计算 孔隙率 $P$ 。从多孔支架的SEM照片中随机选取 100 个 孔, 用Image $\mathrm{J}$ 分析软件统计支架的孔径分布。

$$
P=\frac{W_{2}-W_{1}}{\rho V} \times 100 \%
$$

\section{2 结果与讨论}

\section{1 多孔支架形貌}

图 2 显示, $80{ }^{\circ} \mathrm{C}$ 时制备的复合支架(图 2(a))孔 径较小, 孔分布不均匀, 且存在较多闭合孔, 孔隙 贯通性较差; 而 $90{ }^{\circ} \mathrm{C}$ 发泡制备的支架(图 2(b))中, 孔径分布相对均匀, 主要集中在 300 600 $\mu \mathrm{m}$, 孔形 貌规则, 贯通性高; $100{ }^{\circ} \mathrm{C}$ 和 $110{ }^{\circ} \mathrm{C}$ 制备的支架孔 径较大, 分布不均匀, 大量孔的孔径超过 $1000 \mu \mathrm{m}$ (图 2(c,d))。

本研究中发泡剂水是通过均匀分散在复合材料 中的 DCPD 中的结晶水在一定温度条件下释放获得, 熟化发泡温度对 DCPD/PU 复合支架的孔径大小、 分布和孔隙率大小起主导作用。对比观察支架的孔 隙结构可见, 随着熟化发泡温度的升高, 支架的孔 径增大。DCPD 在温度高于 $75{ }^{\circ} \mathrm{C}$ 后会逐渐失去结 晶水, 在发泡过程中, 均匀分散在 $\mathrm{DCPD} / \mathrm{PU}$ 复合 材料中。从材料内部均匀释放出水, 可与 PU 中的异 氧酸根反应生成 $\mathrm{CO}_{2}$, 实现复合材料体系的 “均相” 自发泡成型。从反应热力学与动力学角度出发, 温
度越高, 反应体系单位时间释放的自由水越多, 生 成的 $\mathrm{CO}_{2}$ 越多，进而越易快速逸出或者合并，从而 导致孔径大小分布的差异(图 2)。当在 $80{ }^{\circ} \mathrm{C}$ 熟化发 泡时, 尽管温度略高于 DCPD 释放结晶水的温度, 但因温度相对较低和 DCPD 包埋于 PU 基质中等原 因, 释放结晶水的速率和量都较小, 相应反应生成 $\mathrm{CO}_{2}$ 也较少, 支架孔径较小, 贯通性差。当采用 $100{ }^{\circ} \mathrm{C}$ 和 $110{ }^{\circ} \mathrm{C}$ 熟化发泡制备支架时，较高的反应温度将 使 DCPD 在较短的时间内释放大量结晶水, 导致 $\mathrm{CO}_{2}$ 快速溢出，制备的支架孔径过大，孔隙分布均 匀性变差, 进而导致力学性能下降。初步预研的力学 性能测试也表明 $100{ }^{\circ} \mathrm{C}$ 和 $110{ }^{\circ} \mathrm{C}$ 制备的 DCPD/PU 复合支架力学性能较差。
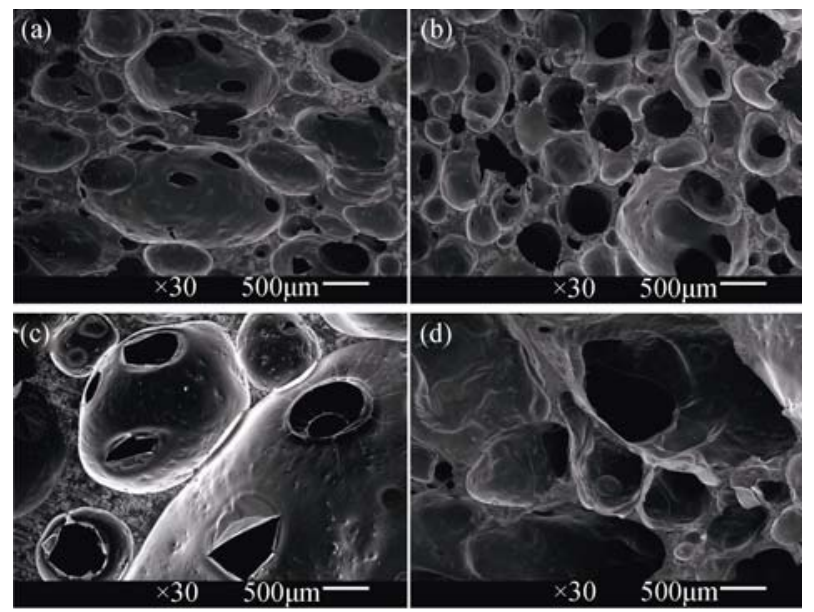

图 2 不同熟化发泡温度下制备的 DCPD/PU 复合多孔支架的 SEM 照片

Fig. 2 SEM micrographs of DCPD/PU composite porous scaffolds formed at different temperature

(a) $80^{\circ} \mathrm{C}$; (b) $90^{\circ} \mathrm{C}$; (c) $100^{\circ} \mathrm{C}$; (d) $110^{\circ} \mathrm{C}$ 
因此，通过改变温度来调节 DCPD 的结晶水 释放，可有效调控复合支架的孔隙率、孔径及孔 隙贯通性。通过对比不同温度下制备的复合支架 的结构和性能, 优选出 $90{ }^{\circ} \mathrm{C}$ 为 DCPD/PU 复合支 架的最佳发泡温度，制备的支架孔径分布均匀、 贯通性好。

$\mathrm{nHA}$ 与自然骨矿物成分相似, 具有良好的生物 相容性和骨传导性，是一种理想的骨修复材料 ${ }^{[16]}$ 。 在优选出的最佳发泡温度 $90{ }^{\circ} \mathrm{C}$ 条件下, 进一步采 用 $\mathrm{nHA}$ 与 $\mathrm{DCPD}$ 的混合粉末与 PU 复合, 制备出 nHA-DCPD/PU 仿生复合多孔支架。图 3 显示，制备 的 $\mathrm{CaP} / \mathrm{PU}$ 复合支架富含贯穿孔，孔形为球形或椭 球形，孔径分布较均匀，主要集中在 100 300 和 300 $700 \mu \mathrm{m}$ (图 3 (d f)), 适合细胞的迁入和增殖 ${ }^{[17]}$ 。未 掺加 nHA 的复合支架(图 3 (a)) 贯通性较好。孔隙率 测试结果显示(表 2)随着 nHA 添加量的增加, DCPD 含量减少，制备的复合支架孔隙率逐渐降低，未添 加 nHA 的支架孔隙率高于添加 nHA 组 $10 \%$ 以上。 主要是因为随着 DCPD 含量降低, 可释放用作发泡 剂的结晶水相应减少，从而导致孔隙率降低。尽管 nHA-DCPD/PU-2 的孔隙率约为 $80.3 \%$, 但依然显著 高于文献报道的在复合材料合成后期加水作为发泡 剂所制备的 $\mathrm{nHA} / \mathrm{PU}$ 多孔支架(其中最大孔隙率约 $63.35 \%)^{[14]}$ 。以上结果表明该方法制备的 $\mathrm{CaP} / \mathrm{PU}$ 支 架的孔结构有利于细胞和骨组织长入, 在骨组织工 程领域具有较大的应用潜力。

\section{$2.2 \mathrm{X}$ 射线衍射分析}

从图 4 可以看出, DCPD/PU、nHA-DCPD/PU-1 和 nHA-DCPD/PU-2 支架的 XRD 谱图中 DCPD 和 nHA 的特征峰位置与 DCPD 和 HA 粉末的衍射峰基本一 致, 强度均弱化。可能是由于 DCPD、nHA 等无机 粒子被 PU 包覆, 使其衍射峰强度减弱。XRD 图谱 也显示支架中 DCPD 峰强度随着 DCPD 含量的减少 而逐渐减弱, 尤以 $2 \theta=12^{\circ}$ 处最为显著。在 $2 \theta=26.6^{\circ}$ 、 $30.2^{\circ} 、 32.9^{\circ}$ 处出现无水磷酸氢钲(DCPA)的衍射峰, 说明 DCPD 在发泡过程中部分或全部脱去结晶水, 以 DCPA 的形式存在于复合支架中，这也将导致 DCPD 衍射峰强度弱化。另外在 $2 \theta=9^{\circ} 、 31.4^{\circ} 、 29.7^{\circ}$ 左右出现在复合多孔支架 XRD 图谱中的衍射峰为 固定 XRD 样品所用橡皮泥(plasticine)衍射峰。由于 多孔支架难以制成粉末，测试时使用橡皮泥将较平 整的支架薄片固定在 XRD 样品台上，在收集 XRD 图谱时，出现了橡皮泥的干扰峰。

\section{3 支架孔隙率与力学性能}

nHA-DCPD/PU-1 和 nHA-DCPD/PU-2 支架的 强度和模量显著高于 $\mathrm{DCPD} / \mathrm{PU}$ 支架(表 2), 如 nHADCPD/PU-1 的压缩强度和模量分别约 1.3 和 1.9 倍 于 $\mathrm{DCPD} / \mathrm{PU}$ 复合支架，主要与 $\mathrm{DCPD} / \mathrm{PU}$ 支架的孔 隙率较高有关。压缩强度等力学性能与多孔支架的 孔隙率密切相关，孔隙率越小，孔壁越厚，多孔支 架的密度越大，抗压能力也越强。
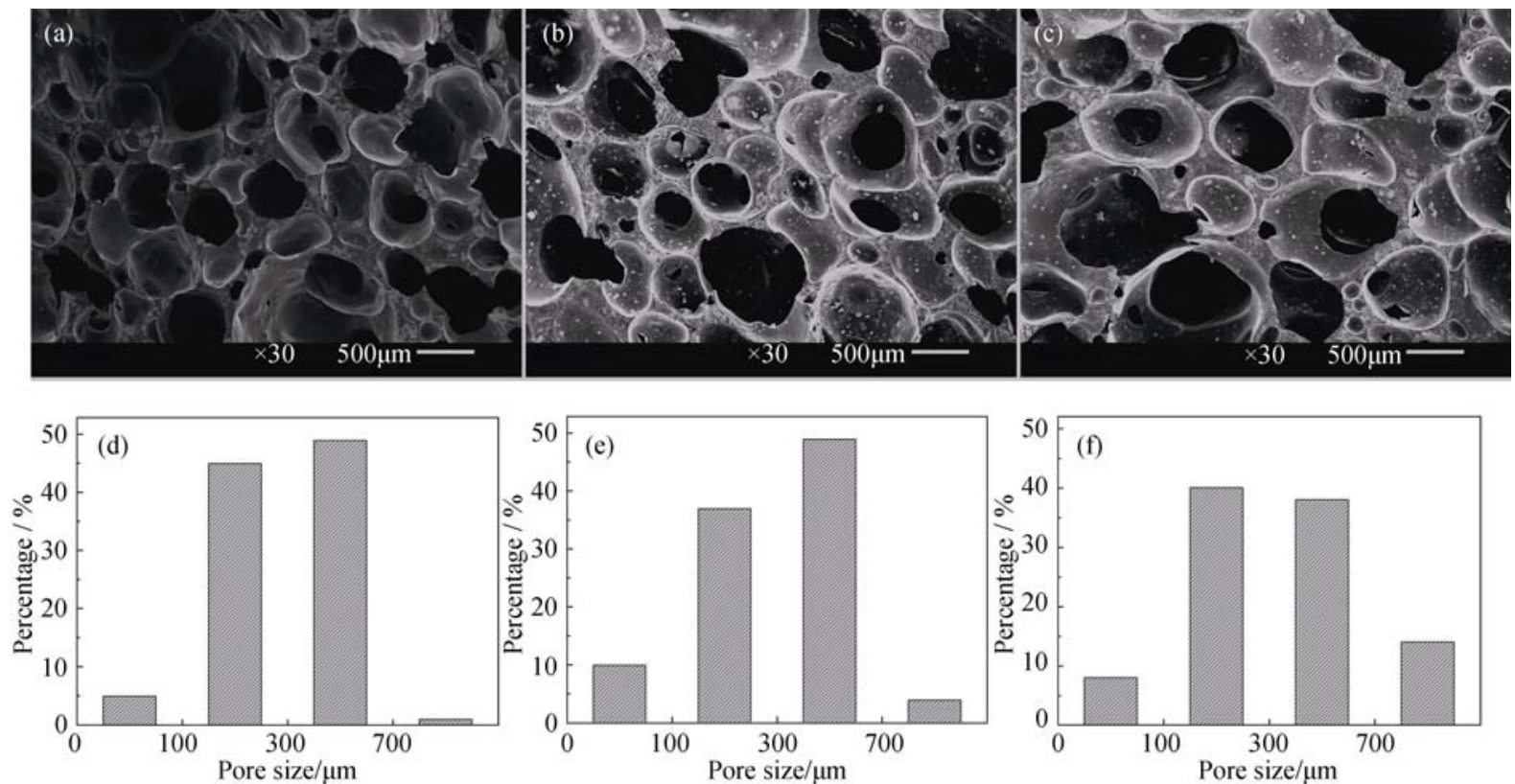

图 $390^{\circ} \mathrm{C}$ 熟化发泡制备的磷酸钲盐/聚氨酯 $(\mathrm{CaP} / \mathrm{PU})$ 多孔支架的 $\mathrm{SEM}$ 照片 $(\mathrm{a} \sim \mathrm{c})$ 及孔径分布图 $(\mathrm{d} \sim \mathrm{f})$

Fig. 3 SEM images (a-c) and pore size distribution (d-f) of CaP/PU porous scaffolds fabricated at $90{ }^{\circ} \mathrm{C}$ (a, d) DCPD/PU; (b, e) nHA-DCPD/PU-1; (c, f) nHA-DCPD/PU-2 
表 $290{ }^{\circ} \mathrm{C}$ 发泡制备的 DCPD/PU、nHA-DCPD/PU-1

和 nHA-DCPD/PU-2 支架的孔隙率和力学性能

Table 2 Porosity and mechanical properties of DCPD/PU, nHA-DCPD/PU-1 and nHA-DCPD/PU-2 composite scaffolds fabricated at $90{ }^{\circ} \mathrm{C}$

\begin{tabular}{cccc}
\hline Composition & Porosity/\% & $\begin{array}{c}\text { Compressive } \\
\text { strength/MPa }\end{array}$ & $\begin{array}{c}\text { Compressive } \\
\text { modulus/MPa }\end{array}$ \\
\hline $\begin{array}{c}\text { DCPD/PU } \\
\text { nHA-DCPD/P } \\
\text { U-1 }\end{array}$ & $92.6 \pm 1.1$ & $1.1 \pm 0.1$ & $31.5 \pm 1.9$ \\
$\begin{array}{c}\text { nHA-DCPD/P } \\
\text { U-2 }\end{array}$ & $82.2 \pm 2.1$ & $1.7 \pm 0.2$ & $58.6 \pm 4.9$ \\
\hline
\end{tabular}

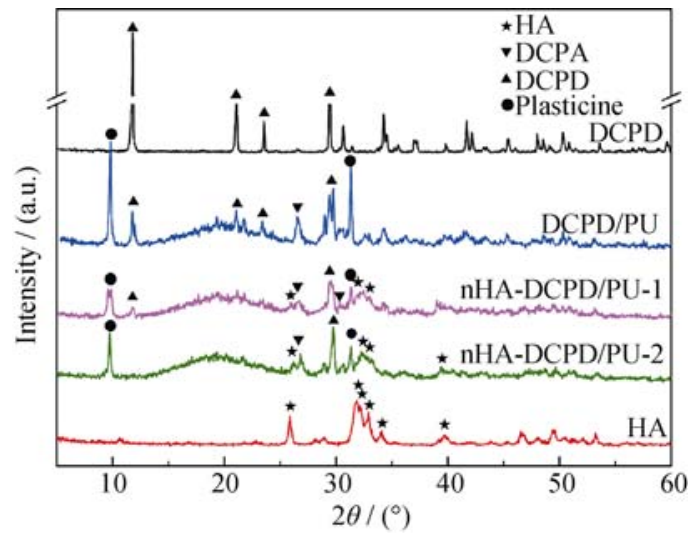

图 $490{ }^{\circ} \mathrm{C}$ 条件下制备的 $\mathrm{CaP} / \mathrm{PU}$ 复合支架、 $\mathrm{nHA}$ 和 DCPD 粉末的 XRD 图谱

Fig. 4 XRD patterns of $\mathrm{CaP} / \mathrm{PU}$ composite scaffolds fabricated at $90{ }^{\circ} \mathrm{C}$, nHA and $\mathrm{DCPD}$ powders

尽管实验优选出 $90{ }^{\circ} \mathrm{C}$ 制备基于 $\mathrm{DCPD} / \mathrm{PU}$ 自 发泡材料体系的支架孔隙率高和孔隙贯通性佳, 但 制备的多孔支架力学性能较差。主要与 $90{ }^{\circ} \mathrm{C}$ 条件 下 PU 的熟化程度不高有关。为进一步提高支架 的力学性能, 支架成型后放入 $110{ }^{\circ} \mathrm{C}$ 烘箱继续熟

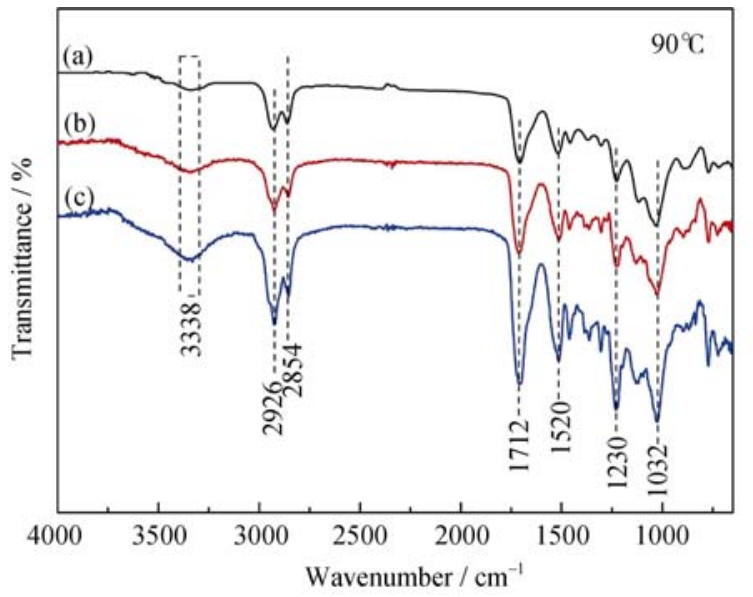

化 $24 \mathrm{~h}$ 。由表 3 可知, 后续 $110{ }^{\circ} \mathrm{C}$ 熟化处理可有效 增强支架力学性能。nHA-DCPD/PU-1 和 nHA$\mathrm{DCPD} / \mathrm{PU}-2$ 复合支架的强度均大幅提升。这可能由 于再熟化提高了支架中 PU 的交联度以及促进了无 机/有机两相的界面结合，进而提高材料的机械性 能。 $\mathrm{DCPD} / \mathrm{PU}$ 支架 $110{ }^{\circ} \mathrm{C}$ 再熟化后力学性能提高 幅度相对较小, 可能因 DCPD/PU 孔隙率高, 孔壁较 薄, 再熟化处理对其力学性能提升作用有限。

\subsection{FTIR 分析}

图 5 的支架红外光谱图显示, $3338 \mathrm{~cm}^{-1}$ 处的 宽峰为硬段 $\mathrm{N}-\mathrm{H}$ 的伸缩振动峰 ${ }^{[18]}, 1712 \mathrm{~cm}^{-1}$ 附近 的吸收峰为氢键化的酰胺碳基伸缩振动峰 ${ }^{[14,19]}$, $1520 \mathrm{~cm}^{-1}$ 左右的峰属于 $\mathrm{C}-\mathrm{N}$ 伸缩振动峰和 $\mathrm{N}-\mathrm{H}$ 弯 曲振动峰的叠加, 位于 $1230 \mathrm{~cm}^{-1}$ 附近的吸收峰则是 酰胺III的振动吸收峰。这些吸收峰的出现表明生成 了氨基甲酸酯交联键。所有图谱中在 $2274 \mathrm{~cm}^{-1}$ 附近 均未见明显的-NCO 的伸缩振动峰, 表明无 $-\mathrm{NCO}$

表 $390{ }^{\circ} \mathrm{C}$ 发泡制备的 DCPD/PU、nHA-DCPD/PU-1 和 nHA-DCPD/PU-2 支架在 $110{ }^{\circ} \mathrm{C}$ 再熟化 $24 \mathrm{~h}$ 后的力学性能 Table 3 Mechanical properties of DCPD/PU, nHA-DCPD/ PU-1 and nHA-DCPD/PU-2 composite scaffolds fabricated at $90{ }^{\circ} \mathrm{C}$ re-cured at $110{ }^{\circ} \mathrm{C}$ for $24 \mathrm{~h}$

\begin{tabular}{cccc}
\hline Composition & $\begin{array}{c}\text { Compressive } \\
\text { strength / } \mathrm{MPa}\end{array}$ & $\begin{array}{c}\text { Compressive } \\
\text { modulus / } \\
\mathrm{MPa}\end{array}$ & $\begin{array}{c}\text { Increase of } \\
\text { compressive } \\
\text { strength after } \\
\text { re-curing / \% }\end{array}$ \\
\hline $\begin{array}{c}\text { DCPD/PU } \\
\text { nHA-DCPD/P } \\
\text { U-1 }\end{array}$ & $2 \pm 0.3$ & $43.8 \pm 2.2$ & 77 \\
$\begin{array}{c}\text { nHA-DCPD/P } \\
\text { U-2 }\end{array}$ & $3.6 \pm 0.4$ & $69.5 \pm 10.2$ & 126 \\
\hline
\end{tabular}

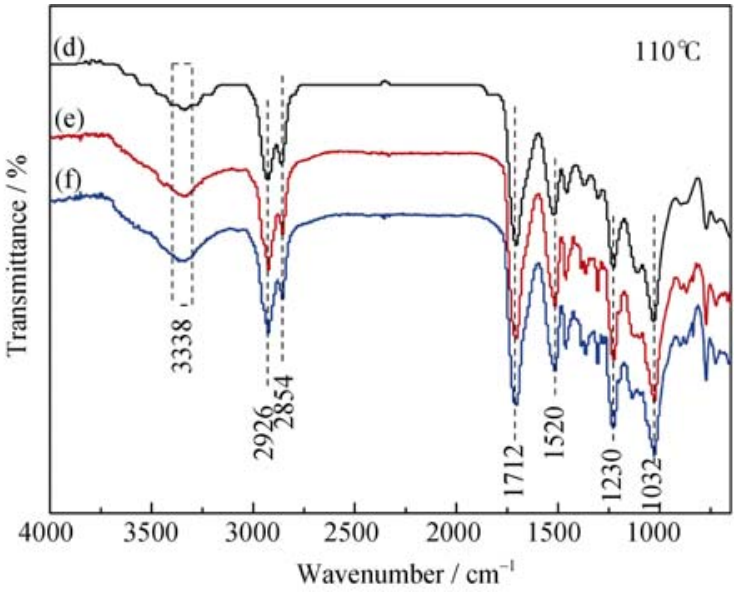

图 $590{ }^{\circ} \mathrm{C}(\mathrm{a} \sim \mathrm{c})$ 发泡和发泡成型后在 $110{ }^{\circ} \mathrm{C}(\mathrm{d} \sim \mathrm{f})$ 再熟化处理的 $\mathrm{CaP} / \mathrm{PU}$ 复合支架的红外光谱图

Fig. 5 FT-IR spectra of CaP/PU composite scaffolds cured at $90{ }^{\circ} \mathrm{C}$ (a-c) and re-cured at $110{ }^{\circ} \mathrm{C}$ (d-f) (a, d) DCPD/PU; (b, e) nHA-DCPD/PU-1; (c, f) nHA-DCPD/PU-2 
残留。除了葛麻油中的羟基与 IPDI 中的-NCO 反应 生成聚氨酯, 体系中存在的大量结晶水也可与 $-\mathrm{NCO}$ 反应, 使-NCO 完全消耗。-NCO 的完全转 化有利于改善聚氨酯基生物材料的生物相容性。 $1032 \mathrm{~cm}^{-1}$ 左右吸收峰可能为 $\mathrm{PO}_{4}{ }^{3-}$ 的非对称伸缩振 动峰与 $\mathrm{C}-\mathrm{O}-\mathrm{C}$ 振动峰的重叠峰。

所有的 PU 基支架在 $1747 \mathrm{~cm}^{-1}$ 附近并未出现明 显的羰基双键 $(\mathrm{C}=\mathrm{O})$ 的伸缩振动特征峰, 可能是极 性较大的酯交换葲麻油基聚氨酯大分子与 DCPD 或 $\mathrm{HA}$ 相容性较好, 以及大量结晶水的存在使体系内 形成大量氢键 $(-\mathrm{OH} \cdots \mathrm{C}=\mathrm{O}$ 和 $-\mathrm{NH} \cdots \mathrm{C}=\mathrm{O})$, 致使自 由羰基减少或消失 ${ }^{[14]}$ 。位于 $1650 \mathrm{~cm}^{-1}$ 附近脲基的 特征峰也未观察到, 可能因为复合材料体系中存在 大量结晶水, 消耗了过量的- $\mathrm{NCO}$, 抑制了-NCO 与 氨基甲酸酯键生成艮基。

相比 $90{ }^{\circ} \mathrm{C}$ 制备的支架, 在 $110{ }^{\circ} \mathrm{C}$ 再熟化处理 后，位于 $1712 \mathrm{~cm}^{-1}$ 附近的氢键化的酰胺碳基伸缩振 动峰强度显著增强, 说明 $110{ }^{\circ} \mathrm{C}$ 熟化处理后聚氨酯 分子链间以及 DCPD 或 HA 与聚氨酯基体分子间形 成了大量的缔合氢键, 增加了高分子链间的交联度 和无机-有机界面的结合强度, 使复合材料的力学 性能得到增强。

\subsection{TG 分析}

图 6 显示, 所有支架的 TG 曲线均出现了两个明 显失重阶段, 第一失重阶段为复合材料中聚氨酯硬 段降解，第二失重阶段对应聚氨酯软段降解 ${ }^{[20]}$ 。

根据 $\mathrm{TG}$ 曲线，以失重 $5 \mathrm{wt} \%$ 对应温度为支架的 初始分解温度来考察支架的热稳定性, 结果如表 4 所示。成型后经 $110{ }^{\circ} \mathrm{C}$ 再熟化处理的支架的初始分 解温度比 $90{ }^{\circ} \mathrm{C}$ 下制备的支架的初始分解温度均高 出 $6{ }^{\circ} \mathrm{C}$ 以上, 表明再熟化处理能增强 $\mathrm{Ca} / \mathrm{PU}$ 复合支

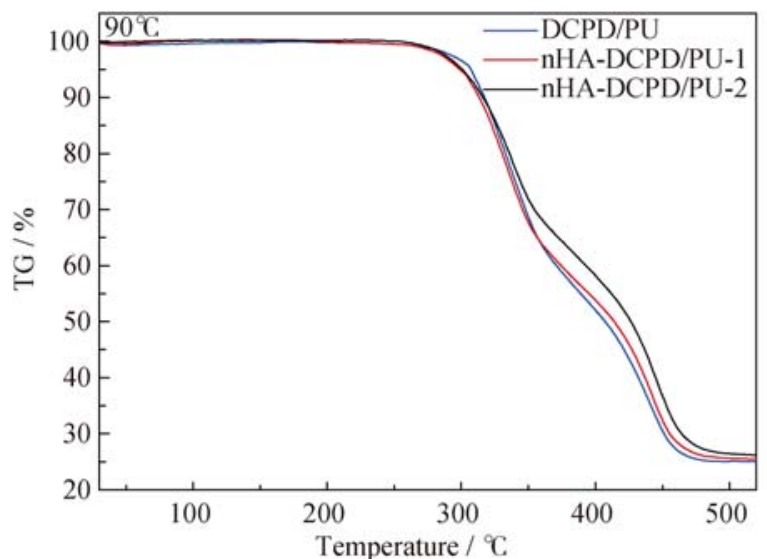

表 $4 \mathrm{CaP} / \mathrm{PU}$ 多孔复合支架的热分析结果 Table 4 Results of thermal analysis of CaP/PU composite scaffolds

\begin{tabular}{|c|c|c|}
\hline & $\begin{array}{c}\text { Onset decompositon } \\
\text { temperature for } \\
\text { samples cured at } \\
90{ }^{\circ} \mathrm{C} /{ }^{\circ} \mathrm{C}\end{array}$ & $\begin{array}{l}\text { Onset decomposition } \\
\text { temperature for samples } \\
\text { recured at } 110{ }^{\circ} \mathrm{C} \text { for } \\
24 \mathrm{~h} /{ }^{\circ} \mathrm{C}\end{array}$ \\
\hline $\mathrm{DCPD} / \mathrm{PU}$ & 309.0 & 316 \\
\hline $\begin{array}{c}\text { nHA-DCPD/ } \\
\text { PU-1 }\end{array}$ & 310.0 & 321 \\
\hline $\begin{array}{c}\text { nHA-DCPD/ } \\
\text { PU-2 }\end{array}$ & 309.6 & 316 \\
\hline
\end{tabular}

架的热稳定性。可能在较高的温度下再熟化提高了 支架中高分子的交联程度。

FTIR 和 TG 分析表明以 DCPD 中结晶水为发 泡剂，在较低的温度下制备的多孔支架可通过成 型后在较高温度下再塑化提高支架中高分子链间 的交联度, 从而提高支架的力学性能, 从实验上 和理论上印证了通过成型后在较高温度下再熟化 处理提高 $\mathrm{Ca} / \mathrm{PU}$ 复合支架力学性能的有效性和可 行性。

\section{3 结论}

1) DCPD 均匀复合在 PU 材料体系中, 一定条 件下释放结晶水与 $\mathrm{PU}$ 中的异氭酸根反应生成 $\mathrm{CO}_{2}$, 可实现复合材料自发泡成型;

2) $90{ }^{\circ} \mathrm{C}$ 使 DCPD 失去结晶水熟化发泡，可制 备出孔隙率高、孔结构均匀、贯通性好的 $\mathrm{CaP} / \mathrm{PU}$ 复合支架;

3) $90{ }^{\circ} \mathrm{C}$ 发泡成型的 $\mathrm{CaP} / \mathrm{PU}$ 支架经 $110{ }^{\circ} \mathrm{C}$ 再 熟化处理，可提高复合支架的力学性能达一倍以 上。该方法制备的孔隙率高、贯通性好、空隙分布

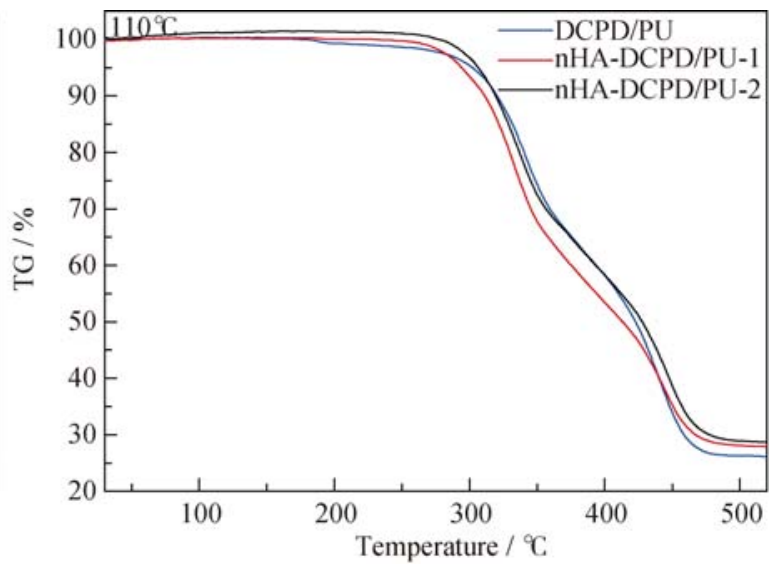

图 $690{ }^{\circ} \mathrm{C}$ 发泡和发泡成型后在 $110{ }^{\circ} \mathrm{C}$ 再熟化处理 $24 \mathrm{~h}$ 的 $\mathrm{CaP} / \mathrm{PU}$ 复合支架的 $\mathrm{TG}$ 曲线

Fig. 6 TG curve of CaP/PU composite scaffolds cured at $90{ }^{\circ} \mathrm{C}$ and re-cured at $110{ }^{\circ} \mathrm{C}$ for $24 \mathrm{~h}$ 
均匀、力学性能好的 $\mathrm{CaP} / \mathrm{PU}$ 复合支架在骨组织工 程领域具有较大的应用潜力。

\section{参考文献:}

[1] REZWAN K, CHEN Q Z, BLAKER J J, et al. Biodegradable and bioactive porous polymer/inorganic composite scaffolds for bone tissue engineering. Biomaterials, 2006, 27: 3413-3431.

[2] JANIK H, MARZEC M. A review: fabrication of porous polyurethane scaffolds. Materials Science and Engineering C, 2015, 48(1): 586-591.

[3] TIENEN T G, HEIJKANTS R, BUMA P, et al. Tissue ingrowth polymers and degradation of two biodegradable porous with different porosities and pore sizes. Biomaterials, 2002, 23: 1731-1738.

[4] KIRDPONPATTARA S, KHAMKERW A, SANCHAVANAKIT $\mathrm{N}$, et al. Structural modification and characterization of bacterial cellulose-alginate composite scaffolds for tissue engineering. Carbohydrate Polymers, 2015, 132(5): 146-155.

[5] YOU F, LI Y B, ZOU Q, et al. Fabrication and osteogenesis of a Porous nanohydroxyapatite/polyamide scaffold with an anisotropic architecture. ACS Biomaterials Science \& Engineering, 2015, 1: 825-833.

[6] TAMIMI F, KUMARASAMI B, DOILLON C, et al. Brushitecollagen composites for bone regeneration. Acta Biomater., 2008, 4: 1315-1321.

[7] MYTHILI J, SUBRAMANIAN M V. Preparation and characterization of two new composites: collagen-brushite and collagen octa-calcium phosphate. Medical Science Monitor: International Medical Journal of Experimental and Clinical Research, 2002, 8(11): $481-487$.

[8] GUELCHER S A. Biodegradable polyurethanes: Synthesis and applications in regenerative medicine. Tissue Engineering Part B: Reviews, 2008, 14(1): 3-17.

[9] MCBANE J E, SHAIFPOOR S, CAI K, et al. Biodegradation and in vivo biocompatibility of a degradable, polar/hydrophobic/ionic polyurethane for tisse engineering applications. Biomaterials, 2011, 32(26): 6034-6044.
[10] GIANNITELLI S M, BASOLI F, MOZETIC P, et al. Graded porous polyurethane foam: a potential scaffold for oro-maxillary bone regeneration. Materials Science and Engineering C, 2015, 51: 329-335.

[11] LIU H H, ZHANG L, ZUO Y, et al. Preparation and characterization of aliphatic polyurethane and hydroxyapatite composite scaffold. Journal of Applied Polymer Science, 2009, 112(5): 2968-2975.

[12] YANG W, BOTH S K, ZUO Y, et al. Biological evaluation of porous aliphatic polyurethane/hydroxyapatite composite scaffolds for bone tissue engineering. Journal of Biomedical Materials Reserach Part A, 2015, 103(7): 2251-2259.

[13] APELT D, THEISS F, ELWARRAK A O, et al. In vivo behavior of three different injectable hydraulic calcium phosphate cements. Biomaterials, 2004, 25: 1439-1451.

[14] LI L M, ZUO Y, DU J J, et al. Structural and mechanical properties of composite scaffolds based on nano-hydroxyapatite and polyurethane of alcoholized castor oil. Journal of Inorganic Materials, 2013, 28(8): 811-817.

[15] GABRIELA C, OCTAVIAN C. Mixed-matrix membranes based on polyurethane containingnanohydroxyapatite and its potential applications. Journal of Applied Polymer Science, 2015, (132)17: 41813 .

[16] WOODARD J R, HILLDORE A J, LAN S K, et al. The mechanical properties and osteoconductivity of hydroxyapatite bone scaffolds with multi-scale porosity. Biomaterials, 2007, 28: 45-54.

[17] HULBerT S F, YOUNG F A, MATHEWS R S, et al. Potential of ceramic materials as permanently implantable skeletal prostheses. Journal of Biomedical Materials Research, 1970, 4(3): 433-456.

[18] TERAMOTO N, SAITOH Y, TAKAHASHI A, et al. Biodegradable polyurethane elastomers prepared from isocyanate-terminated poly(ethylene adipate), castor oil, and glycero. Journal of Applied Polymer Science, 2010, 115(6): 3199-3204.

[19] MISHRA A K, CHATTOPADHYAY D K, SREEDHAR B. FT-IR and XPS studies of polyurethane-urea-imide coatings. Progress in Organic Coatings, 2006, 55(3): 231-243.

[20] MOTHE C G, ARAUJO C R. Properties of polyurethane elastomers and composites by thermal analysis. Thermochimicaacta, 2000, 357(1): 321-325. 